\title{
The relationship between sleep quality and psychological distress and job burnout among Chinese psychiatric nurses
}

\author{
Bin WANG ${ }^{1}$, Qinghua $\mathrm{LU}^{2} *$, Feifei $S \mathrm{UN}^{2}$ and Rui $\mathrm{ZHANG}^{3}$ \\ ${ }^{1}$ Psychological Department of Shandong Provincial Hospital, China \\ ${ }^{2}$ Nursing Department of Shandong Mental Health Center, China \\ ${ }^{3}$ School of Nursing, Shandong First Medical University \& Shandong Academy of Medical Sciences, China
}

Received December 2, 2020 and accepted May 19, 2021

Published online in J-STAGE September 27, 2021

DOI https://doi.org/10.2486/indhealth.2020-0249

\begin{abstract}
This cross-sectional study aimed to investigate the sleep quality of psychiatric nurses in China and explore the risk factors affecting it. This study used the stratified random sampling method. The general data questionnaire was conducted using the 10-item Kessler Psychological Distress Scale (K10), the Maslach Burnout Inventory (MBI), and the Pittsburgh Sleep Quality Index (PSQI) were used to investigate the prevalence and risk factors of sleep quality among $\mathbf{8 1 2}$ psychiatric nurses in China. There were statistically significant differences in sleep quality among different shift frequency. Surveys demonstrated that sleep quality among psychiatric nurses was positively correlated with psychological distress and job burnout. Multiple logistics regression analysis showed that high psychological distress (odds ratio, $O R=0.907, p<0.001,95 \%$ confidence interval, $C I=0.885-0.931)$, high emotional exhaustion $(\mathrm{OR}=0.946, p<0.001,95 \% \mathrm{CI}=0.921-$ 0.972), low depersonalization $(\mathrm{OR}=1.061, p=0.004,95 \% \mathrm{CI}=1.019-1.104)$, and low personal accomplishment $(\mathrm{OR}=0.972, p=0.018,95 \% \mathrm{CI}=\mathbf{0 . 9 4 9 - 0 . 9 9 5 )}$ were the contributing factors of sleep quality. Future studies should investigate effective measures to relieve psychological distress and alleviate burnout, particularly for psychiatric nurses with poor sleep quality.
\end{abstract}

Key words: Psychiatric nurse, Sleep quality, Psychological distress, Burnout, China

\section{Introduction}

Sleep is one of the most important physiological needs of human beings. Poor sleep quality refers to the physical and psychological states that trigger a series of detrimental effects because of the abnormal amount of sleep or low sleep

*To whom correspondence should be addressed.

E-mail address: 1172869296@qq.com

(C2021 National Institute of Occupational Safety and Health quality ${ }^{1)}$. Sleep disorders may lead to reduced immunity, reduced adaptive ability, anxiety, depression, and other physical and mental disorders ${ }^{2}$. Previous studies have demonstrated a significant discrepancy between actual and expected sleep duration, with insomnia being common among nurses, particularly psychiatric nurses in China ${ }^{3}$. Negative emotions, such as anger and irritability, among nurses may be caused by poor sleep quality ${ }^{4}$. At the same time, negative emotions could results in reduced quality of work ${ }^{5}$. Recently, numerous studies have demonstrated that poor sleep quality among nurses can lead to health prob- 
lems with a high risk of adverse events ${ }^{6}$, which may influence the continuity of care delivered to patients and threaten patients' safety ${ }^{7)}$. With the worldwide increase in life expectancy and incidence of chronic diseases, such as mental illness, the demand for nurses has also grown steadily, while the number of available nurses is unable to meet the growing demand ${ }^{8)}$. Studies have shown that nurses' health was negatively affected by insufficient sleep, resulting in problems such as diabetes, hypertension, and heart disease ${ }^{9)}$. With the increase in work pressure, sleep disorders have become a prominent public health concern. Therefore, the impact of sleep quality merits further investigation.

Studies have identified a number of factors associated with sleep, including antenatal psychological distress, stress, and lifestyle behaviors ${ }^{10}$. A survey of undergraduate students revealed that anxiety and psychological distress were significant independent predictors of poor sleep quality ${ }^{11)}$. A study of German adolescents suggested that poor sleep quality was associated with psychological distress ${ }^{12)}$. Studies have reported that dissatisfaction with sleep patterns was associated with emotional exhaustion and a high level of depersonalization among nursing workers. Studies showed that severe burnout was closely related to psychological stress ${ }^{13}$. Burnout affects mental and physical health, it may cause depression, anxiety, and alcohol dependence ${ }^{14}$. Studies have shown that psychological distress can affect sleep quality, such causing as insomnia and short periods of sleep (fewer than 6 hours), which were reported by individuals with high levels of Burnout Syndrome ${ }^{15}$. However, other studies found that average daily sleep was not affected by burnout ${ }^{16)}$. In addition, job stress is strongly associated with depression ${ }^{17}$. Individual sleep quality can be disturbed and sleep disorders may be caused or aggravated by psychological disturbances, such as anxiety and depres$\operatorname{sion}^{18)}$. Psychiatric nurses are a special professional group who served for the people with mental disorders. They often face situations involving patient abuse, attacks, escape, suicide, self-harm, and other emergencies ${ }^{19)}$. Psychiatric nurses have heavy work burdens, high risk, and psychological pressure ${ }^{20)}$. Some studies showed that the incidence of sleep problems among psychiatric nurses was $21.6 \%$, which affected their mental health ${ }^{21)}$, aggravating their sleep problems. To date, there has been no extensive research on the relationship between psychological distress, burnout, and sleep quality from the perspective of psychiatric nurses.

This study aimed to examine the correlation between sleep and psychological distress and burnout among psy- chiatric nurses in order to provide a theoretical basis for nursing management, improve sleep quality, and ensure patient safety.

\section{Materials and Methods}

\section{Participants}

According to the statistics of Shandong Province of China, there were approximately 8,000 registered psychiatric nurses in Shandong Province as of 2019. According to the finite population sampling formula, set $\mathrm{K}=1.96, p=0.5$, $\alpha=0.05$, the number of samples investigated should be over 367. This study selected 904 nurses. The convenience sampling method was applied in this study. There are six natural regions in Shandong Province: northwest Shandong, southwest Shandong, south Shandong, north Shandong, middle Shandong and Shandong peninsula. A total of 904 clinical nurses were randomly selected from a tertiary psychiatric hospital in each region. Inclusion criteria included nurse managers and clinical psychiatric nurses with the National Qualified Certificate of Practice Nursing who had worked for more than one year and were voluntarily investigated. Exclusion criteria included nursing interns, nurses who engaged in advanced studies, and nurses who had been on sick leave for more than three months. After obtaining the consent of the ethics committee of the investigated unit, the researchers consulted the director of the nursing department and the full-time investigator of the investigated unit, who distributed paper questionnaires to the participants. The methods and important points were explained to the participants. The participants filled out the questionnaires anonymously and returned the results within one week. A total of 904 questionnaires were distributed, 812 of which were valid, and the effective rate was $89.82 \%$.

\section{Ethical considerations}

The questionnaires strictly followed the principle of informed consent, and the study was approved by the Ethics Committee of Shandong Mental Health Center. The investigators introduced the purpose and the basic information. During the whole study process, the privacy and anonymity of participants were fully protected. The respondents gave their written informed consent to participate in this study.

\section{Measurement of psychological distress}

The 10-items Kessler Psychological Distress Scale (K10) includes 10 items established by Kessler and Mroczek $^{22)}$ and is widely used to assess individual's psychological distress. The 10 items were rated on a 5-point 
Likert from 1 (hardly) to 5 (very much). Higher scores indicated higher levels of psychological distress. A total score of greater than 16 indicated psychological distress ${ }^{23}$. The reliability and internal validity have been verified ${ }^{24)}$, the Cronbach's $\alpha$ in the present study was 0.930 .

The Maslach Burnout Inventory was mainly used to evaluate job burnout. The 22 items of the scale included three aspects: emotional exhaustion, depersonalization, and low personal accomplishment ${ }^{25}$. The Chinese version comprised 22 items, involves emotional exhaustion (nine items), depersonalization (five items), and personal accomplishment (eight items) ${ }^{26}$. All items were scored on a scale of 0 to 6 . Emotional exhaustion consisted of nine items, measuring feelings of being emotionally overextended and exhausted by one's work. The scoring range was $0-54$ points. Depersonalization included five items, which were mainly used to evaluate an unfeeling and impersonal response toward recipients of one's service, care treatment, or instruction by work pressure. The score ranged from 0 to 30 points. Items in the above two aspects were scored positively, that is, the higher the score, the more serious the job burnout. Personal accomplishment included eight items. Main evaluation caused by view of competence and successful achievement in one's work, with the score range of $0-48$ points. An entry in this category was scored in reverse, meaning the lower the score, the greater the job burnout. The Cronbach's $\alpha$ was $0.872,0.801$ and 0.819 for the subscales in this study.

The Pittsburgh Sleep Quality Index (PSQI) was a measure of subjective Sleep Quality in the last month. Including the subjective sleep quality, sleep latency, sleep continuity, habitual sleep efficiency, sleep disorders and hypnotic drugs, daytime function seven factors, each factor was rated on a 4-point Likert from 0 (no difficulties) to 3 (very difficult). The cumulative score of each factor was the total score of the Pittsburgh Sleep Quality Index Scale, with the total score ranging from 0 to 21 . The higher the score, the worse the sleep quality. Scores greater than 5 indicated poor sleep quality. Sufferers were defined as those who scored more than 5 points, while those who scored less than or equal to 5 points were defined as non-sufferers. The Chinese version of the PSQI was considered a reliable and valid measure of sleep quality ${ }^{27,28)}$. The Cronbach's $\alpha$ was 0.839 .

\section{Statistical analysis}

Data input and statistical analysis were carried out using SPSS 21.0 statistical software and $p<0.05$ was considered statistically significant. The continuous variables were ex- pressed as mean and standard deviation. The categorical variables were expressed by frequency and percentage and the univariate analysis was performed by t-test or single-factor chi-square test to assessed the sample characteristics and relationships among variables. Pearson correlation was used to analyze the relationship between sleep quality, psychological distress, and burnout. Multivariate logistic regression analysis was conducted, with sleep quality as the dependent variable, while the item work shift with statistical significance in univariate analysis, Psychological Distress (K10), and the Maslach Burnout Inventory (MBI) were independent variables.

\section{Results}

\section{Descriptive and correlational analysis}

The 812 psychiatric nurses were between 18 and 58 years old, with an average age of $32.69 \pm 8.07$ years. Years of Psychiatric nursing were between one and 40. Median working years were 6 (p $25=3$ years, p $75=15$ years). Overall, 208 respondents were men (25.6\%) and 604 were women $(74.4 \%)$. Regarding education, 341 (42.0\%) had college degree or below, and $471(58.0 \%)$ with bachelor's degree or above. In addition, 630 were married (77.6\%), 182 were not married $(22.4 \%)$. Regarding professional titles, 283 were nurses (34.9\%), 294 were junior nurses (36.2\%), 193 were senior nurses (23.8\%), 42 were associate superintendent nurses $(5.2 \%)$. The average number of night shift days per week was $2.20 \pm 1.19$.

Characteristics of the participants and the comparisons of the scores on PSQI are presented in Table 1. The average PSQI score was $9.10 \pm 2.78$, which included 431 participants $(53.08 \%)$ with a PSQI $>5$. Poor sleep quality was statistically correlated with work shift $(p<0.05)$. There was a significant difference in poor sleep quality among different work shifts, while there were no significant differences based on age, sex, marital status, educational level, professional title, income, and years of psychiatric nursing.

The mean psychological distress score was 17.88 ( $\mathrm{SD}=6.57$, range $10-47)$ and the PSQI scores $<5$ were $46.92 \%$. The emotional exhaustion score was 20.77 ( $\mathrm{SD}=8.71$, range $1-47)$. The depersonalization score was 9.17 ( $\mathrm{SD}=5.63$, range $0-28$ ). The low personal accomplishment score was 18.90 ( $\mathrm{SD}=7.35$, range $0-46)$. Compared with the non-sufferer group (PSQI scores $<5$ ), the mean psychological distress score of the sufferer group was 24.31 ( $\mathrm{SD}=8.15$, range $10-50, \mathrm{t}=-12.427, p=0.000)$. Participants who suffered from poor sleep quality exhibited significantly higher mean scores for psychological distress $(\mathrm{t}=-12.427$, 
Table 1. Characteristics of the participants and the comparisons of the scores on PSQI

\begin{tabular}{|c|c|c|c|c|c|c|}
\hline Variables & & $\mathrm{N}$ & $\mathrm{PSQI}>5$ & Prevalence (\%) & $\chi^{2}$ & $p$ \\
\hline \multirow[t]{3}{*}{ Age } & $<30$ years & 379 & 198 & 52.24 & 0.307 & 0.858 \\
\hline & $30-39$ years & 254 & 135 & 53.15 & & \\
\hline & $\geq 40$ years & 179 & 98 & 54.75 & & \\
\hline \multirow[t]{2}{*}{ Sex } & Male & 208 & 105 & 50.48 & 0.758 & 0.384 \\
\hline & Female & 604 & 326 & 53.97 & & \\
\hline \multirow[t]{2}{*}{ Marital status } & married & 630 & 327 & 51.90 & 1.556 & 0.212 \\
\hline & single & 182 & 104 & 57.14 & & \\
\hline \multirow[t]{2}{*}{ Educational level } & Junior school or under & 341 & 182 & 53.37 & 0.020 & 0.887 \\
\hline & Bachelor's degree or above & 471 & 249 & 52.87 & & \\
\hline \multirow[t]{4}{*}{ Professional title } & nurse & 283 & 147 & 51.94 & 3.771 & 0.287 \\
\hline & junior nurse & 294 & 158 & 53.74 & & \\
\hline & senior nurse & 193 & 109 & 56.48 & & \\
\hline & Associate superintendent nurse & 42 & 17 & 40.48 & & \\
\hline \multirow[t]{3}{*}{ Income } & $\leq 3,000$ yuan & 243 & 127 & 52.26 & 0.567 & 0.753 \\
\hline & $3,000-5,000$ yuan & 384 & 209 & 54.43 & & \\
\hline & $>5000$ yuan & 185 & 95 & 51.35 & & \\
\hline \multirow[t]{2}{*}{ Work shift } & day shift only & 130 & 54 & 41.54 & 8.277 & 0.004 \\
\hline & three-shift rotation & 682 & 377 & 55.28 & & \\
\hline \multirow[t]{3}{*}{ Years of working } & $1-4$ years & 297 & 147 & 49.49 & 2.444 & 0.295 \\
\hline & $5-9$ years & 203 & 111 & 54.68 & & \\
\hline & $\geq 10$ years & 312 & 173 & 55.45 & & \\
\hline
\end{tabular}

Note: 'three-shift rotation' is a fixed rotating shift schedule, which consists of an 8-hour day shift, an 8-hour swing shift and an 8-hour night shift.

$p<0.001$ ), emotional exhaustion ( $\mathrm{t}=-8.929, p<0.001)$, depersonalization ( $\mathrm{t}=-4.644, p<0.001)$, and low personal accomplishment $(\mathrm{t}=-3.660, p<0.001)$ (Table 2$)$.

The psychological distress of psychiatric nurses was positively correlated with subjective sleep quality $(r=0.456$, $p<0.01)$, sleep latency $(r=0.371, p<0.01)$, sleep continuity $(r=0.318, p<0.01)$, habitual sleep efficiency $(r=0.158$, $p<0.01)$, sleep disorders $(r=0.420, p<0.01)$, hypnotic drugs $(r=0.212, P<0.01)$, and daytime function $(r=0.447, p<0.01)$.
Emotional exhaustion was positively correlated with subjective sleep quality $(r=0.366, p<0.01)$, sleep latency $(r=0.309, p<0.01)$, sleep continuity $(r=0.235, p<0.01)$, habitual sleep efficiency $(r=0.129, p<0.01)$, sleep disorders ( $r=0.242, p<0.01)$, hypnotic drugs $(r=0.169, p<0.01)$, and daytime function $(r=0.392, p<0.01)$. Depersonalization was positively correlated with subjective sleep quality ( $r=0.170, p<0.01)$, sleep latency $(r=0.170, p<0.01)$, sleep continuity $(r=0.077, p<0.05)$, sleep disorders $(r=0.113$, 
$p<0.01)$, hypnotic drugs $(r=0.134, p<0.01)$, and daytime function $(r=0.222, p<0.01)$. Low personal accomplishment was positively correlated with subjective sleep quality $(r=0.116, p<0.01)$, sleep latency $(r=0.111, p<0.01)$, sleep disorders $(r=0.136, \quad p<0.01)$, and daytime function $(r=0.124, p<0.01)$ (Table 3).

To quantify the influence of sleep quality on psychiatric nurses, multiple logistic regression model was used to analyze the factors influencing poor sleep quality (Table 4). The risk factors for poor sleep quality among psychiatric nurses were high psychological distress $(\mathrm{OR}=0.907$, $p<0.001,95 \% \mathrm{CI}=0.885-0.931$ ), high emotional exhaustion $(\mathrm{OR}=0.946, p<0.001,95 \% \mathrm{CI}=0.921-0.972)$, low depersonalization $(\mathrm{OR}=1.061, p=0.004,95 \% \mathrm{CI}=1.019$ 1.104), and high levels of low personal accomplishment $(\mathrm{OR}=0.972, p=0.018,95 \% \mathrm{CI}=0.949-0.995)$.

\section{Discussion}

Current situation of sleep quality among psychiatric nurses

In this study, the average PSQI score was $(9.10 \pm 2.78)$, which included 431 participants $(53.08 \%)$ with a PSQI $>5$. The average PSQI score was higher than the normal population and nurses in other specialized hospitals. For instance, a survey of 636 nurses from seven hospitals of different levels in Spain showed $6.80 \pm 3.39^{29)}$. A study in Japan found that 109 nurses in general hospitals had a sleep score of $6.0 \pm 2.1^{30}$. This may be associated with a strong hidden risk of mental diseases. Patients often have violent attacks and other emergencies under the influence of mental symptoms, such as hallucinations and delusions. Therefore, the sleep quality of nurses is affected to some extent. It may also be related to the differences in the region and environment of the nurses under investigation. In addition, univariate analysis of socio-demographic factors showed that poor sleep quality had no significant difference based on age, gender, marital status, educational level, professional title, income, and working years. However, it was significantly associated with work shift. Shift-work nurses were more likely to experience sleep disturbance than day nurses. This study showed that $55.28 \%$ of shift-work nurses had sleep disturbances compared to $41.54 \%$ among day nurses. This may be related to fewer nurses working at night. Most psychiatric hospitals in this study assigned 2 nurses to work at night. The ratio of nurses to patients was significantly lower at night than in the daytime. In case of a violent attack, escape, suicide, or other emergencies, there may not have been enough nurses to deal with the situation. Therefore, nurses working at night were highly stressed. As such, shift-work nurses were more likely to be in a state of stress than daytime nurses. At the same time, shift-work forcefully disrupts the normal sleep-wake cycle. As such, sleep is more likely to be disrupted in the daytime, potentially leading to shorter sleep periods ${ }^{31}$. This was in line with previous studies indicating that specific characteristics of shiftwork nurses can lead to poor sleep quality ${ }^{32,33)}$. A previous study of sleep quality among shift nurses found that it was difficult for nurses to stay awake for $36.0 \%$ of shift time, which doubled their risk of errors ${ }^{34)}$. Therefore, shift-work psychiatric nurses require special attention.

Correlation between sleep quality and psychological distress among psychiatric nurses

This study demonstrated a statistically significant difference between the scores of psychological distress and sufferers and non-sufferers of poor sleep. Psychological distress was positively correlated with scores of sleep dimensions. That is, higher psychological distress was correlated with worse sleep, such as insomnia and early waking ${ }^{10)}$. Psychological distress referred to an emotional state characterized by depression and anxiety. Studies have shown that psychological distress has a negative impact on sleep quality ${ }^{35}$. There is a two-way interaction between sleep quality, anxiety, and depression, and insomnia is an

Table 2. Relationship between psychological distress, job burnout and poor sleep quality among psychiatric nurses

\begin{tabular}{lcccc}
\hline Factor & $\begin{array}{c}\text { Sufferers } \\
\text { PSQI }>\mathbf{5}\end{array}$ & $\begin{array}{c}\text { Non-sufferers } \\
\text { PSQI } \leq \mathbf{5}\end{array}$ & & $p$ \\
& Mean \pm SD & Mean \pm SD & & \\
\cline { 2 - 4 } & $24.31 \pm 8.15$ & $17.88 \pm 6.57$ & -12.427 & 0.000 \\
\hline Psychological distress (K10) & $26.37 \pm 9.10$ & $20.77 \pm 8.71$ & -8.929 & 0.000 \\
Emotional exhaustion & $10.97 \pm 5.36$ & $9.17 \pm 5.63$ & -4.644 & 0.000 \\
Depersonalization & $20.67 \pm 6.21$ & $18.90 \pm 7.35$ & -3.660 & 0.000 \\
Low personal accomplishment & & & & \\
\hline
\end{tabular}



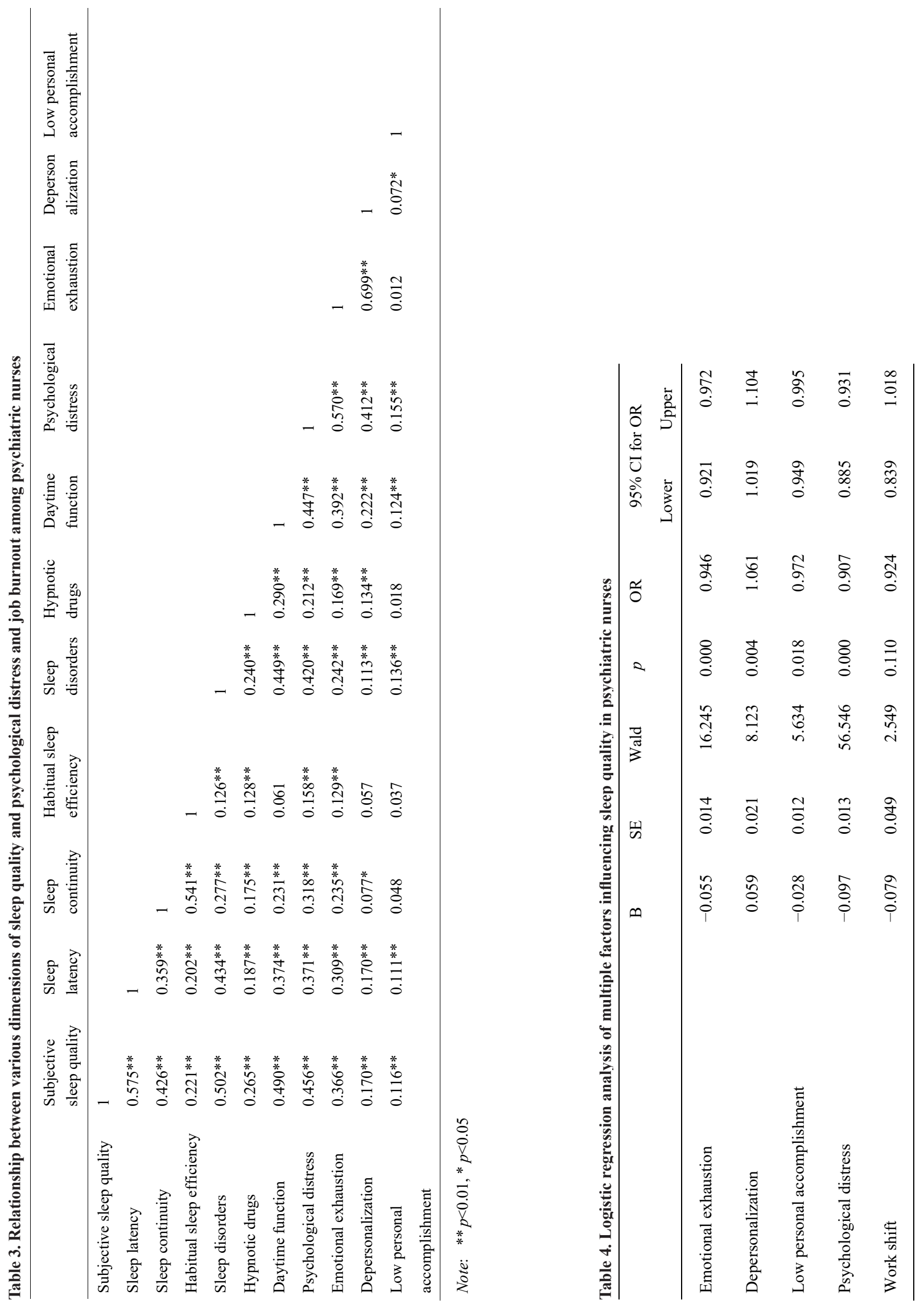
important predictor of depression recurrence and uncured depression ${ }^{36}$. This may be related to anxiety, depression, and other psychological factors, which may extend fast wave sleep, cause sleep disorders, and activate the body's stress system. Remaining in this state for an extended period may lead to anxiety, depression, and other psychological problems. In addition, these emotional problems may alternatively increase the incidence of sleep disorder. This is in line with previous research ${ }^{10)}$.

This study showed a positive correlation between job burnout and sleep disorders. The logistic regression analysis showed that psychological distress, low personal accomplishment, emotional exhaustion, and depersonalization were associated with the development of poor sleep quality, which is consistent with previous studies ${ }^{37,38)}$. This may be associated with higher risks in psychiatric care, as nurses often face patients with violent attacks, suicide, self-injury, and other emergencies ${ }^{20)}$. However, nurses have fewer requirements for difficult nursing operation skills. To some extent, this may hinder the work enthusiasm of psychiatric nurses, resulting in feelings of low personal accomplishment and negative emotions, such as anxiety and depression. This further leads to the disharmony of the nurse-patient relationship and a decline of work efficiency and quality, which may in turn affect the attitude of psychiatric nurses toward work, causing apathy and insensitivity to emotional exhaustion. In addition, burnout may be a risk factor for poor sleep. Exposure to long-term job stress and insufficient rest can affect individuals' health ${ }^{39)}$. A lack of adequate rest leads to depletion of the energy reserves of individuals exposed to chronic job stress. According to previous research, long term exposure to job stress and decreased ability to recover from burnout may lead to cognitive deficits and dysregulation of the HPA axis ${ }^{40)}$, which in turn affect sleep. On the other hand, effective sleep can refresh brain function, regulate physiological function, and regulate and reorganize mood, behavior, cognition, and memory. Sleep disorders are more likely to cause individuals to suffer from depression, anxiety, inattention, and other symptoms of burnout, interacting in a vicious circle.

This study had some limitations. First, this study used a convenience sample method to recruit psychological nurses from six tertiary hospitals in Shandong Province. Consequently, the generalization may be limited. Future studies should include more provinces in China. Second, this study used only self-reported subjective measures and all data were collected by self-report questionnaires, which may affect the results. Third, as this was a cross-sectional survey, no causal relationships could be established.

\section{Conclusions and implications}

This study demonstrated a high incidence of sleep disorders among psychiatric nurses. Psychological distress and job burnout were correlated with sleep disorders and were the risk factors of sleep disorders. Therefore, psychiatric nursing managers should focus on the improvement of nurses' occupational burnout. In order to improve the overall level of mental health among psychiatric nurses, group intervention strategies, such as mindfulness and interpersonal relationship therapy, should be adopted to cultivate positive mood and behavior patterns, reduce anxiety, depression, and other psychological problems, alleviate mental fatigue, and improve sleep quality.

\section{References}

1) Dong H, Zhang Q, Sun Z, Sang F, \& Xu Y (2017) Sleep disturbances among Chinese clinical nurses in general hospitals and its influencing factors. BMC Psychiatry 17, 241.

2) Du Y, Xu L, Qi H (2013) Quality of sleep and related factors among junior middle school students of grade three in Gaomi city. Chin J Public Health 8, 1215-7.

3) An FR, Qi YK, Zeng JY, Ding YM, Chiu HF, Ungvari GS, Newhouse R, Yu DSF, Lai KYC, Xiang YT (2016) The prevalence of insomnia, its demographic correlates, and treatment in nurses working in Chinese psychiatric and general hospitals. Perspect Psychiatr Care 52, 88-94.

4) Salari N, Khazaie H, Hosseinian-Far A, Ghasemi H, Mohammadi M, Shohaimi S, Daneshkhah A, KhalediPaveh B, Hosseinian-Far M (2020) The prevalence of sleep disturbances among physicians and nurses facing the COVID-19 patients: a systematic review and meta-analysis. Global Health 16, 92.

5) Fang R, Li X (2015) A regular yoga intervention for staff nurse sleep quality and work stress: a randomised controlled trial. J Clin Nurs 24, 3374-9.

6) Rathore H, Shukla K, Singh S, Tiwari G (2012) Shift work-problems and its impact on female nurses in Udaipur, Rajasthan India. Work 41 Suppl 1, 4302-14.

7) Surani S, Murphy J, Shah A (2007) Sleepy nurses: are we willing to accept the challenge today? Nurs Adm Q 31, 146-51.

8) Schilgen B, Nienhaus A, Handtke O, Schulz H, Mosko M (2017) Health situation of migrant and minority nurses: a systematic review. PLoS One 12, e0179183.

9) Beebe D, Chang JJ, Kress K, Mattfeldt-Beman M (2017) Diet quality and sleep quality among day and night shift nurses. J Nurs Manag 25, 549-7.

10) Almojali AI, Almalki SA, Alothman AS, Masuadi EM, Alaqeel MK (2017) The prevalence and association of stress with sleep quality among medical students. JEGH 7, 169-74. 
11) Seun-Fadipe CT, Mosaku KS (2017) Sleep quality and psychological distress among undergraduate students of a Nigerian university. Sleep Health 3, 190-4.

12) Sampasa-Kanyinga H, Chaput JP, Hamilton HA, Colman I (2018) Bullying involvement, psychological distress, and short sleep duration among adolescents. Soc Psychiatry Psychiatr Epidemiol 53, 1371-80.

13) Shenoi AN, Kalyanaraman M, Pillai A, Raghava PS, Day S (2018) Burnout and psychological distress among pediatric critical care physicians in the United States. Crit Care Med 46, 116-22.

14) Ramírez MR, Otero P, Blanco V, Ontaneda MP, Díaz O, Vázquez FL (2018) Prevalence and correlates of burnout in health professionals in Ecuador. Compr Psychiatry 82, 73-83.

15) Vidotti V, Ribeiro RP, Galdino M, Martins JT (2018) Burnout Syndrome and shift work among the nursing staff. Revista Latino-Americana De Enfermagem 26, e3022.

16) Mendelsohn D, Despot I, Gooderham PA, Singhal A, Redekop GJ, Toyota BD (2019) Impact of work hours and sleep on well-being and burnout for physicians-in-training: the resident activity tracker evaluation study. Med Educ 53, 306-15.

17) Wang SM, Lai CY, Chang YY, Huang CY, Zauszniewski JA, Yu CY (2015) The relationships among work stress, resourcefulness, and depression level in psychiatric nurses. Arch Psychiatr Nurs 29, 64-70.

18) Tu CY, Chou YH, Lin YH, Huang WL (2019) Sleep and emotional disturbance in patients with non-dialysis chronic kidney disease. J Formos Med Assoc 118, 986-94.

19) Jiang H, Zhong G, Lu Q (2018) Analysis on the present situation and influencing factors of work stress of in psychiatric nurses. Journal of Qilu Nursing 24, 82-4.

20) Lin HS, Probst JC, Hsu YC (2010) Depression among female psychiatric nurses in southern Taiwan: main and moderating effects of job stress, coping behaviour and social support. J Clin Nurs 19, 2342-54.

21) Lu Q, Zhong G (2014) Investigation on the sleep quality of nurses of a certain mental hospital at grade III level and analysis of the influencing factors. Journal of Qilu Nursing 21, 10-2.

22) Kessler RC, Green JG, Gruber MJ, Sampson NA, Bromet E, Cuitan M, Zaslavsky AM (2010) Screening for serious mental illness in the general population with the K6 screening scale: results from the WHO World Mental Health (WMH) survey initiative. Int J Methods Psychiatr Res 19 Suppl 1, 4-22.

23) Andrews G, Slade $T$ (2001) Interpreting scores on the Kessler Psychological Distress Scale (K10). Aust N Z J Public Health 25, 494-7.

24) Tian X, Gao Q, Li G, Zou G, Liu C, Kong L, Li P (2016) Resilience is associated with low psychological distress in renal transplant recipients. Gen Hosp Psychiatry 39, 86-90.

25) Champion DF, Westbrook BW (1984) Maslach Burnout Inventory. Meas Eval Couns Dev 17, 100-2.
26) Hua YU (2007) The application of Chinese version of Maslach Burnout Inventory in Chinese nurses. J Nurs Sci 22, 50-2.

27) Li G, Kong L, Zhou H, Kang X, Fang Y, Li P (2016) Relationship between prenatal maternal stress and sleep quality in Chinese pregnant women: the mediation effect of resilience. Sleep Med 25, 8-12.

28) Zhang J, Lu C, Tang J, Qiu H, Liu L, Wang S, Wang A, Zhang T, Zhao S, Jiang Y, Zhao C, Hu B (2008) A crosssectional study of sleep quality in people aged 18 years or over in Shandong Province. Chin J Psychiatry 41, 97-101.

29) Gómez-García T, Ruzafa-Martínez M, Fuentelsaz-Gallego C, Madrid JA, Rol MA, Martínez-Madrid MJ (2016) Nurses' sleep quality, work environment and quality of care in the Spanish National Health System: observational study among different shifts. BMJ Open 6, e012073.

30) Morimoto H, Tanaka H, Ohkubo R, Mimura M, Ooe N, Ichikawa A, Yukitoshi H (2016) Self-help therapy for sleep problems in hospital nurses in Japan: a controlled pilot study. Sleep Biol Rhythms 14, 177-85.

31) Wright KPJr, Bogan RK, Wyatt JK (2013) Shift work and the assessment and management of shift work disorder (SWD). Sleep Med Rev 17, 41-54.

32) Giorgi F, Mattei A, Notarnicola I, Petrucci C, Lancia L (2018) Can sleep quality and burnout affect the job performance of shift-work nurses? A hospital crosssectional study. J Adv Nurs 74, 698-708.

33) Kecklund G, Axelsson J (2016) Health consequences of shift work and insufficient sleep. BMJ 355, i5210.

34) Li Z, Sun DM, Li CB, Tao MF (2016) Influencing factors for sleep quality among shift-working nurses: a crosssectional study in China using 3-factor Pittsburgh Sleep Quality Index. Asian Nurs Res (Korean Soc Nurs Sci) 10, 277.

35) John-Henderson NA, Williams SE, Brindle RC, Ginty AT (2018) Changes in sleep quality and levels of psychological distress during the adaptation to university: the role of childhood adversity. Br J Psychol 109, 694-707.

36) Zhang X, Zhang X, Yu J (2020) [Analysis of sleep quality and characteristics in patients with depression accompanied with different degrees of anxiety]. Cardiovasc Dis J Integr Tradit Chin West Med 35, 71+77 (in Chinese).

37) Felder JN, Laraia B, Coleman-Phox K, Bush N, Suresh M, Thomas M, Adler N, Epel E, Prather AA (2018) Poor sleep quality, psychological distress, and the buffering effect of mindfulness training during pregnancy. Behav Sleep Med 16, 611-24.

38) Zhou H, Li W, Ren Y (2020) Poor sleep quality of third trimester exacerbates the risk of experiencing postnatal depression. Psychol Health Med 25, 229-38.

39) Yang T, Qiao Y, Xiang S, Li W, Gan Y, Chen Y (2019) Work stress and the risk of cancer: a meta-analysis of observational studies. Int J Cancer 144, 2390-400.

40) Guglielmi O, Jurado-Gamez B, Gude F, Buela-Casal G (2014) Job stress, burnout, and job satisfaction in sleep 
apnea patients. Sleep Med 15, 1025-30. 